\title{
Effect of Physical Activity on the Lesion Repair Process in the Gastrocnemius Muscle of Rats
}

\author{
Eli Luis Namba, Thiago Otávio Nawick, Mariele Jung Thome, Paula Cristina Trevilatto, Maria \\ Angela Naval Machado, Luciana Reis Azevedo-Alanis, Antônio Adilson Lima, Aline Cristina \\ Batista Rodrigues Johann, Edvaldo Antônio Ribeiro Rosa, Sérgio Aparecido Ignácio and Ana Maria \\ Trindade Grégio*
}

\author{
Pontifícia Universidade do Paraná - School of Healt and Biosciences- Dentistry Course, Biosciences Area and \\ Universidade Federal do Paraná - Dentistry Course, Brazil
}

\begin{abstract}
The present study aimed to evaluate the effect of a progressive load of physical activity on a musculoskeletal injury in rats. Sixty-four rates were divided into two groups: experimental group (EG), which underwent physical activity (swimming) with a progressive load, and the control group (CG), which was not submitted to this program. The training was carried out according to an adapted version of the Gonçalves (1999) swimming system. Injuries were caused to the gastrocnemic muscle by inducing $40 \% \mathrm{NaOH}$. On days 2, 7, 14, and 21 after inducing muscle injury, the animals from both EC and CG were sacrificed. The injured area was removed and processed. The neutrophils, macrophages, lymphocytes, and plasmocytes were quantified by hematoxylin and eosin (H\&E) stain. The percentage of the area of type I and type III collagen were quantified by a histological cut stained with picrosirius red stain (PSR) and submitted to polarization. A greater number of macrophages ( $\mathrm{GE}=19.98 \pm 4.39 ; \mathrm{GC}=2.48 \pm 1.00 ; \mathrm{p}=0.006)$ and lymphocytes $(\mathrm{GE}=2.12 \pm 0.82 ; \mathrm{GC}=0.06 \pm 0.82 ; \mathrm{p}=0.037)$ after 2 days, and of macrophages $(\mathrm{GE}=15.74 \pm 3.00 ; \mathrm{GC}=6.02 \pm 1.95 ; \mathrm{p}=0.007)$ and lymphocytes $(\mathrm{GE}=2.01 \pm 0.78 ; \mathrm{GC}=0.14 \pm 0.09 ; \mathrm{p}=0.044)$ after 7 days, could be observed in the $\mathrm{EG}$ when compared to the $\mathrm{CG}$; however, the opposite was true for neutrophils $(\mathrm{GE}=48.12 \pm 17.04 ; \mathrm{GC}=105.54 \pm 12.25 ; \mathrm{p}=0.005)$. After 14 days, a smaller quantity of neutrophils $(\mathrm{GE}=32.70 \pm 10.26 ; \mathrm{GC}=90.96 \pm 17.62 ; \mathrm{p}=0.044)$ and a larger quantity of plasmocytes $(\mathrm{GE}=9.06 \pm 3.84 ; \mathrm{GC}=0.68 \pm 0.53 ; \mathrm{p}=0.028$ ) could be observed in the $\mathrm{EG}$ as compared to the CG. A greater area of type III collagen could be observed in the EG when compared to the $\mathrm{CG}$ over a 14 -day period $(\mathrm{GE}=44.90 \pm 16.15 ; \mathrm{GC}=0.74 \pm 0.40$; $\mathrm{p}=0.000)$ and a 21 -day period $(\mathrm{GE}=13.19 \pm 9.09 ; \mathrm{GC}=1.02 \pm 0.94 ; \mathrm{p}=0.029)$, whereas the opposite could be observed for type I collagen. The physical activity promoted an increase in the deposition of type III collagen in the muscle injury. This activity accelerated the repair process after 14 days, possibly moderated by the larger number of inflammatory cells, while after 21 days, the lesion presented a lesser resistance.
\end{abstract}

Keywords: Progressive load, inflammation, repair process, collagen.

\section{INTRODUCTION}

Prior studies have shown the beneficial effects of physical activity on the lesion repair process in osteochondral defects of equine joints [1], in the Achilles tendon of rabbits [2], and in cutaneous wound healing [3, 4]. Gregory et al. (1995) [5] verified the effect of physical activity (swimming and running) in the skeletal muscles of rats after injury (not in ulcerations) and concluded that physical activity is beneficial to muscle repair. However, no study to date has analyzed the cell events and the collagen deposition in muscle ulcers of animals that had undergone physical activity. This evaluation is highly important, given that muscle injuries are part of the day-to-day routine of athletes who undergo high physical impact, which

*Address correspondence to this author at the Pontifícia Universidade do Paraná - School of Health and Biosciences- Dentistry Course, Biosciences Area, Brazil; Tel: +55 41 32711637;

Fax: +55 41 32711405; E-mail: ana.gregio@pucpr.br corresponds to $10 \%$ to $55 \%$ of all injuries suffered by athletes while practicing sports, the lesion repair process being essential to their return to sports activities [6].

The lesion repair process after injury passes through many different stages. The inflammation phases begin as soon as the tissue is damaged and as inflammatory cells begin to interact with the injured area, contributing to tissue repair by producing pro-inflammatory molecules, as well as by stimulating the proliferation of blood vessels and fibroblasts and the synthesis of the cell matrix [7]. Physical activity, when performed in parallel with an inflammatory process, tends to favor the tissue healing process. [8] During physical activity, some changes in the cells involved in the inflammatory process can be observed. Physical activity increases most types of circulating leucocytes (e.g. lymphocytes, basophils, and eosinophils) [9]. Lymphocytes seem to be predisposed to apoptosis when submitted to moderate physical activity but rise in migration at greater intensities, which suggests the migration of these cells away from the blood vessels in post-physical activity evaluations 
[10]. By contrast, macrophages appear to be stimulated during physical stress, and their functions are improved, in turn increasing phagocytosis [11]. In addition, physical activity induces an increase in the macrophage chemotaxis index [12]. As regards collagen, an earlier increase in the diameter of collagen fibers was observed, which suggests that a faster maturation occurred in the tendon submitted to physical activity [2].

Considering that physical activity can interfere positively in the lesion repair process, increasing the number and function of inflammatory cells and favoring the collagen deposition, the hypothesis formulated by the present study is that injured rats submitted to physical activity would present a greater inflammatory response and a greater collagen deposition, capable of accelerating the lesion repair process. In this sense, the pioneer results of this study help to fill a gap in the literature on the issue.

\section{MATERIALS AND METHOD}

The present study was approved by the Animal Research Ethics Committee from the Pontific Catholic University of Paraná (CEUA/PUCPR), under protocol number 297/07. The sample consisted of 64 male Wistar rats, with an age of 50 days, weighing between 250 and 300 grams. The animals were fed rations and received water ad libitum, respecting a photoperiod of 12 hours of light and 12 hours of darkness.

The animals were divided into two groups: a) Experimental Group (EG), with 32 animals with muscle injuries and which had previously practiced physical activity, and b) Control Group (CG), with 32 sedentary animals with muscle injuries. Each animal group was further subdivided into four subgroups $(n=8)$ of $2,7,14$, and 21 days after the induction of the muscle injury. The lesion repair process was evaluated with no therapeutic interference.

The CG animals, although not submitted to physical activity (swimming), were placed in water and immediately removed in an attempt to simulate the neurophysiological stimulus induced in the EG.

The EG animals swam in a scheme of 5 days of physical activity and 2 days of rest/week, according to the methodology set forth by Gonçalves and Luciano (1999) [8]. The swimming sessions occurred at 8:00 am in an asbestos recipient $(100 \mathrm{~cm} \times 70 \mathrm{~cm} \times 60 \mathrm{~cm})$ with a water column of 40 $\mathrm{cm}$ at $32^{\circ} \mathrm{C}$.

In the first five days undergoing physical activity (swimming), the animals adapted to the water environment, beginning with 10-minute intervals and increased 10 minutes each day. As of the sixth day, a load equal to $5 \%$ of the animal's body weight was attached each day to the animal's thorax using a rubber band. On the eleventh day, a muscle injury was induced and the animals continued their training for $2,7,14$, and 21 days after the induction of the muscle injury, that is, totaling $13,18,25$, and 32 days of physical activity (swimming), respectively.

After having been anesthetized by an $80 \mathrm{mg} / \mathrm{kg}$ sodium thiopental ${ }^{\circledR}$ intraperitoneal injection (Cristália, SP, Brazil), the subcutaneous induction of an injury was caused to the right gastrocnemic muscle with $80 u \mathrm{~L}$ of $40 \% \mathrm{NaOH}$. After
$2,7,14$, and 21 days, as mentioned above, the CG and EG animals were sacrificed by means of an intraperitoneal injection with an overdose of anesthesia (sodium thiopental, $150 \mathrm{mg} / \mathrm{Kg}$ ).

The injured area was removed, fixed in $10 \%$ formaldehyde, and processed. Histological cuts of $5 \mu \mathrm{m}$ in thickness were stained with hematoxylin and eosin (H\&E) and Picrosirius Red (PSR). On the slides stained with H\&E, images of the entire extension of the ulcer were captured by an Olympus ${ }^{\circledR}$ BX50 microscope (Olympus America, Inc., Lake Success, NY) coupled to a Sistema Dinolite ${ }^{\circledR}$ microcamera (DI-Noc Chemical Arts, Inc, Honeoye, NY) with a final magnification of 400x. The images were analyzed using the Software Image Tool 3.00 for Windows (Health Science, University of Texas, Austin, TX). The number of neutrophils, macrophages, lymphocytes, and plasmocytes were quantified. To accomplish this, the criteria of this evaluation were based on the morphological aspects, considering the oval-shaped cell plasmocytes with basophilic cytoplasm, with spherical and eccentric nuclei, with chromatin in particles. The neutrophils were identified by the polyform format of the nuclei. Lymphocytes were considered the cells with small, round, and hyperchromatic nuclei, revealing a lack of cytoplasm. By contrast, the macrophages presented ample, hyperchromatic, and oval or bean-shaped nuclei, with a greater quantity of cytoplasm [13].

From the PSR stained cuts, two images were randomly captured using an Olympus BX-50 microscope (Olympus America, Inc., Lake Success, NY) with an Olympus ${ }^{\circledR}$ polarized lens (Olympus America, Inc., Lake Success, NY) and U-Pot coupled to a Dinolite ${ }^{\circledR}$ microcamera (Dino-Lite, AnMo Electronics Corp., Hsinchu, Taiwan) at a magnifycation of 200X. The images were analyzed in an Image ProPlus 4.5 morphometry program (Media Cybernetics, Silver Spring. MD) to obtain the percentage of the area of type I and type III collagen. Type I collagen was defined as that which presented a reddish-orange color, while type III collagen was defined was that which presented a greenishyellow color [14]. The final values were determined by the median of the fields.

This study employed the SPSS ${ }^{\circledR} 18.0$ statistics package (SPSS Inc., Chicago, IL). Also employed were the Kolmogorov-Smirnov normality test and the Levene test to check the homogeneity of the sample. Thus, the two-way ANOVA and the Games-Howell tests were chosen, and the 95\% statistical significance level was adopted.

\section{RESULTS}

A greater number of macrophages and lymphocytes could be observed in the EG as compared to the CG after 2 days, and of macrophages, neutrophils, and lymphocytes after 7 days. However, after 14 days, a lesser number of neutrophils and a greater number of plasmocytes could be observed in the EG as compared to the CG (Table 1).

A greater area of type III collagen could be observed in the EG as compared to the CG over a 14-day period 
Table 1. Average number of neutrophils, macrophages, lymphocytes, and plasmocytes in the CG and EG after 2, 7, 14, and 21 days.

\begin{tabular}{|c|c|c|c|c|c|c|c|c|c|c|c|c|}
\hline Time (Days)/ & \multicolumn{3}{|c}{2} & \multicolumn{3}{c|}{7} & \multicolumn{3}{c|}{14} & \multicolumn{2}{c|}{21} \\
\hline Group/ Cells & GC & GE & p & GC & GE & p & GC & GE & p & GC & GE & p \\
\hline \hline NEUTROPHILS & $6.02 \pm 1.96$ & $15.75 \pm 3.01$ & 0.69 & $19.35 \pm 3.97$ & $16.24 \pm 1.89$ & 0.00 & $14.57 \pm 3.89$ & $8.61 \pm 2.83$ & 0.00 & $19.99 \pm 4.39$ & $12.75 \pm 6.73$ & 0.12 \\
\hline MACROPHAGES & $2.48 \pm 1.00$ & $19.98 \pm 4.39$ & 0.01 & $6.02 \pm 1.95$ & $15.74 \pm 3.00$ & 0.01 & $19.35 \pm 3.97$ & $16.24 \pm 1.88$ & 0.67 & $14.56 \pm 3.88$ & $8.61 \pm 2.82$ & 0.17 \\
\hline LYMPHOCYTES & $0.06 \pm 0.82$ & $2.12 \pm 0.81$ & 0.04 & $0.14 \pm 0.09$ & $2.01 \pm 0.78$ & 0.04 & $0.98 \pm 0.66$ & $1.93 \pm 0.26$ & 0.14 & $1.32 \pm 0.40$ & $0.79 \pm 0.38$ & 0.41 \\
\hline PLASMÓCYTOS & $0.12 \pm 0.15$ & $1.11 \pm 0.78$ & 0.29 & $0.04 \pm 0.07$ & $8.67 \pm 4.04$ & 0.07 & $0.68 \pm 0.53$ & $9.06 \pm 3.84$ & 0.03 & $0.49 \pm 0.42$ & $1.95 \pm 3.02$ & 0.94 \\
\hline
\end{tabular}

In bold, statistically significant differences; Legend: $\mathbf{C G}=$ control group; $\mathbf{E G}=$ experimental group; $\mathbf{M}=$ median; $\mathbf{S D}=$ standard deviation

Table 2. Percentage of the area of type I and III collagens in the EG and CG groups, stratified by time periods of 2, 7, 14, and 21 days.

\begin{tabular}{|c|c|c|c|c|}
\hline \multirow{2}{*}{ Collagen Type } & \multirow{2}{*}{ Time } & EG & CG & \multirow{2}{*}{$p$ value } \\
\hline & & $\mathrm{M} \pm \mathrm{SD}$ & $\mathrm{M} \pm \mathrm{SD}$ & \\
\hline \multirow{4}{*}{ Type I } & 2 & $98.98 \pm 0.04$ & $98.34 \pm 1.66$ & 0.299 \\
\hline & 7 & $98.21 \pm 2.33$ & $98.83 \pm 1.21$ & 0.997 \\
\hline & 14 & $55.10 \pm 16.15$ & $99.26 \pm 0.40$ & 0.000 \\
\hline & 21 & $86.81 \pm 9.09$ & $98.98 \pm 0.94$ & 0.029 \\
\hline \multirow{4}{*}{ Type III } & 2 & $0.02 \pm 0.04$ & $1.66 \pm 1.66$ & 0.299 \\
\hline & 7 & $1.79 \pm 2.33$ & $1.17 \pm 1.21$ & 0.997 \\
\hline & 14 & $44.90 \pm 16.15$ & $0.74 \pm 0.40$ & 0.000 \\
\hline & 21 & $13.19 \pm 9.09$ & $1.02 \pm 0.94$ & 0.029 \\
\hline
\end{tabular}

In bold, statistically significant differences; Legend: $\mathbf{C G}=$ control group; $\mathbf{E G = e x p e r i m e n t a l ~ g r o u p ; ~} \mathbf{M = m e d i a n ;} \mathbf{S D = s t a n d a r d ~ d e v i a t i o n}$

$(\mathrm{GE}=44.90 \pm 16.15 ; \mathrm{GC}=0.74 \pm 0.40 ; \mathrm{p}=0.000)$ and a 21 -day period $(\mathrm{GE}=13.19 \pm 9.09 ; \mathrm{GC}=1.02 \pm 0.94 ; \mathrm{p}=0.029)$, whereas the opposite could be observed for type I collagen (Table 2 , Fig. 1).

\section{DISCUSSION}

Physical activity impacted the lesion repair process, leading to a reduction in the number of neutrophils; an increase in the number of macrophages, lymphocytes, and plasmocytes; as well as a greater area of type III collagen. The greater number of inflammatory cells verified in the present study, with the exception of neutrophils, can be justified by the exacerbation of the initial injury, aggravated by the physical activity in the injured muscle group. This greater number of inflammatory cells may well have favored a greater deposition of type III collagen between 14 and 21 days.

Neutrophils, a kind of granulocyte, are the first cells of defense to reach the inflamed site, and their function is that of phagocytosis. However, a large number of neutrophils can inhibit the lesion repair process $[15,16]$, and the prolonged presence of these cells can provoke a vicious cycle in which the cytokines and proteolytic fragments of the extracellular matrix are released locally to intensify leukocytosis and, possibly, to break down tissues [17]. In this sense, the greater number of neutrophils verified after 7 days in the EG may have indirectly favored the greater deposition of type III collagen after 14 days, considering that, since these cells were identified in a smaller quantity, possibly a lesser quantity of enzymes were also released, in turn promoting a lesser breakdown of the tissues, favoring progress in the lesion repair process. Kudaeva et al. (2012) [18] observed that regular physical activity (swimming) of moderate intensity over a long period promotes a decrease of granulocytes in the peripheral blood. These authors postulate that the reduced relative content of granulocytes in the peripheral blood can be associated with the development of inflammation in muscle tissue, which is characteristic of active working muscles, the migration of granulocytic cells towards the site of inflammation, and their rapid death in these areas. This finding justifies, at least partially, the lower number of neutrophils observed in the present study in the EG after 7 and 14 days. Nevertheless, the results of the present study run in direct contrast with the presence of neutrophils observed by Godbout et al. (2006) [19] in an acute injury caused to the tendon after physical activity. The data regarding the effect of physical activity on the immune system of humans, as well as on experimental animals, are contradictory. This effect depends on the type, duration, and intensity of loads and immune parameters studied. In many cases, the suppression of immune processes was also observed [18]. 


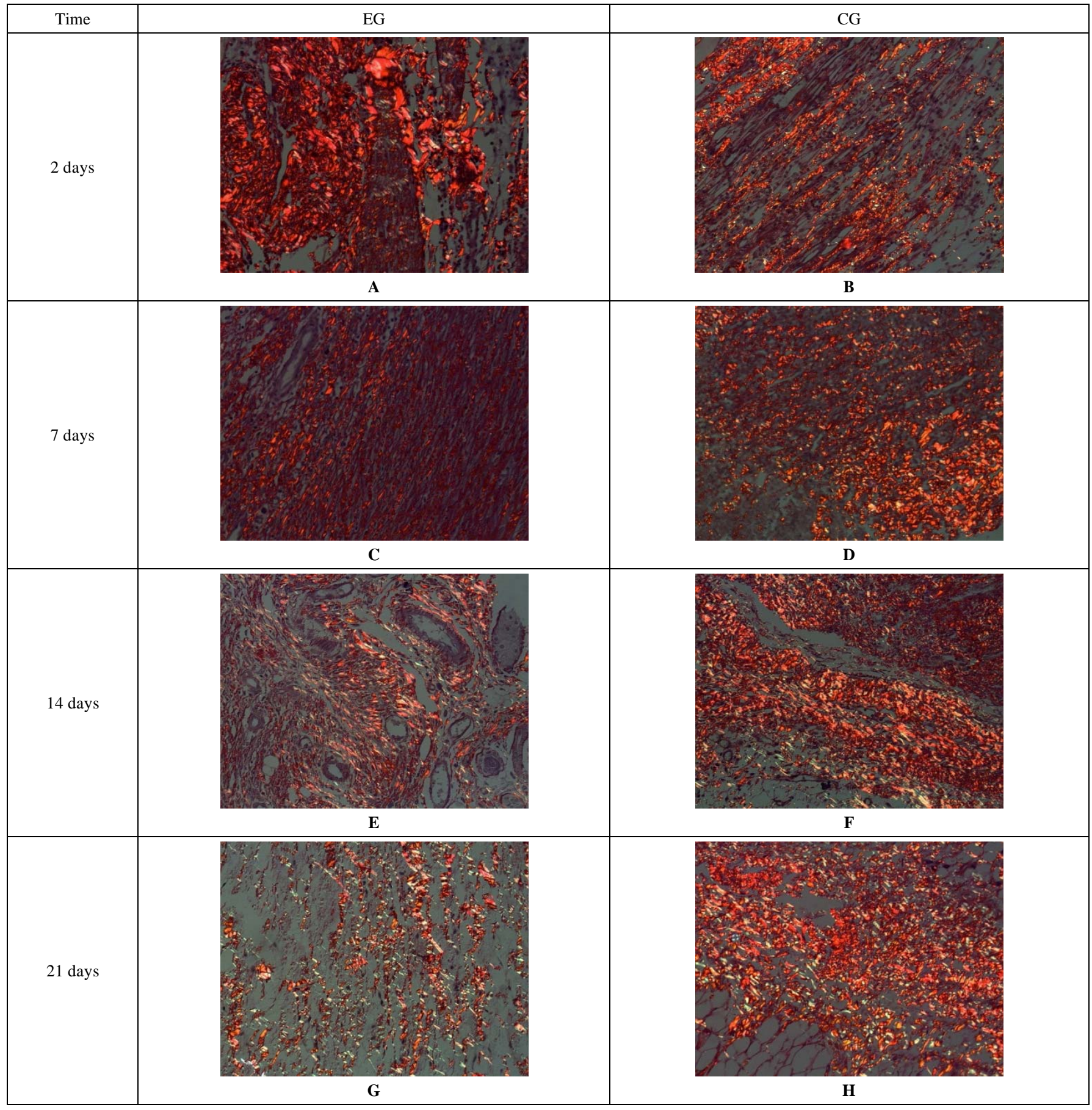

Fig. (1). Photomicrography of Injury of the Gastrocnemius Muscle - A greater area of type III collagen was observed in the EG as compared to the CG in the periods of 14 days ( $\mathbf{E}$ and $\mathbf{F}$, respectively) and 21 days ( $\mathbf{G}$ and $\mathbf{H}$, respectively), while the opposite was observed for type I collagen. For the remaining days, no differences were observed between the groups (SR, 200x).

Macrophages play an important role in the lesion repair process, as they are capable of promoting angiogenesis and tissue repair. The effect of the macrophages on the extracellular matrix presents a nature that is both destructive, secreting metalloproteinase and degrading the interstice, as well as inductive of the lesion repair process, phagocytizing tissue remains and antigens and contributing indirectly to the formation of the extracellular matrix and to its stabilization by stimulating other cells to proliferate and release components from the cell matrix [20]. Macrophages release a number of growth-promoting factors and cytokines (e.g.
FGF, IGF-1, TGF- $\beta 1$ ) that both recruit and activate the fibroblasts that will secrete cell matrix molecules, such as collagen, to begin the lesion repair process [17]. In this manner, this larger number of macrophages may have favored an acceleration in the deposition of type III collagen after 14 days. Godbout et al. (2006) [19] also reported an increase in the number of macrophages in an acute injury of the tendon after physical activity.

Kudaeva et al. (2012) [18] observed that regular physical activity (swimming) of moderate intensity over a long period promotes an increase in the relative number of lymphocytes 
and a rise in plasma cells in the peripheral blood. Increased levels of estrogen and IL- 6 during physical activity can lead to the polyclonal activation of B-lymphocytes, which can be confirmed by the appearance of plasma cells in the peripheral blood of mice [18]. This increase in the number of lymphocytes and plasma cells was also observed in the present study. The polyclonal activation of B cells can be observed, together with a reduction in the intensity of the immune response. Hence, the phenomenon of the polyclonal activation of B-lymphocytes during exercise may well be a cause of immunosuppression under these conditions [18]. It is possible that, if the animals form the present study had been evaluated over a longer period of time, this immunosuppression may well have been detected.

Findings from Gregory et al. (1995) [8] verified that animals injured when submitted to physical activity (swimming) presented no statistically significant differences over the periods of 2, 4, 16, and 32 days regarding the percentage of collagen, which was evaluated by the Gomori's trichrome stain. In the present study, according to the employed technique, a reduction in the type I collagen (mature) could be observed in the EG; however, a larger deposition of type III collagen (immature) could be observed in the periods of 14 and 21 days. The type III collagen appears as thin fibers. These are different from type I collagen, which appears in fiber bundles in bands with a high tensile strength, which, in the present study, confirms the lesser lesion resistance after 21 days.

\section{CONCLUSION}

The physical activity promotes an increase in the deposition of type III collagen in the muscle injury, and this activity aided the repair process after 14 days, possibly moderated by the larger number of inflammatory cells, while after 21 days, the lesion presented a lesser resistance.

\section{CONFLICT OF INTEREST}

The authors confirm that this article content has no conflicts of interest.

\section{ACKNOWLEDGEMENT}

Declared none.

\section{REFERENCES}

[1] Todhunter RJ, Freeman KP, Yeager AE, Lust G. Effects of exercise and polysulfated glycosaminoglycan on the development of osteoarthritis in equine carpal joints with osteochondral defects. Vet Surg 1993; 22: 330-42.

[2] Date T. The influence of exercise in the healing of the rabbit Achilles tendon. Nihon Seikeigeka Gakkai Zasshi 1986; 60: 44954.

[3] Keylock KT, Vieira VJ, Wallig MA, DiPietro LA, Schrementi M, Woods JA. Exercise accelerates cutaneous wound healing and decreases wound inflammation in aged mice. Am J Physiol Regul Integr Comp Physiol 2008; 294: R179-84.

[4] Pence BD, Dipietro LA, Woods JA. Exercise speeds cutaneous wound healing in high-fat diet-induced obese mice. Med Sci Sports Exerc 2012; 44: 1846-54.

[5] Gregory TM, Heckmann RA, Francis RS. The effect of exercise on the presence of leukocytes, erythrocytes and collagen fibers in skeletal muscle after contusion. J Manipulative Physiol Ther 1995; 18: 72-8.

[6] Järvinen TA, Järvinen TL, Kääriäinen M, Kalimo H, Järvinen M. Muscle injuries: biology and treatment. Am J Sports Med 2005; 33: 745-64.

[7] Midwood KS, Williams LV, Schwarzbauer JE. Tissue repair and the dynamics of the extracellular matrix. Int J Biochem Cell Biol 2004; 36:1031-7.

[8] Gonçalves AL, Luciano E. Inflammatory response in Wistar rats submitted to the physical activity. Rev Bras Atividade Fís Saúde 1999; 4: 39-46.

[9] Schwindt CD, Zaldivar F, Wilson L, et al. Do circulating leucocytes and lymphocyte subtypes increase in response to brief exercise in children with and without asthma? Br J Sports Med 2007; 41: 34-40.

[10] Navalta JW, Lyons S, Prestes J, Arnett SW, Schafer M, Sobrero GL. Exercise intensity and lymphocyte subset apoptosis. Int J Sports Med 2013; 34: 268-73.

[11] Rosa LFPBC, Vaisberg MW. Exercise influence on immune response. Rev Bras Med Esporte 2002; 8: 167-72.

[12] Ortega E, Forner MA, Barriga C. Exercise-induced stimulation of murine macrophage chemotaxis: role of corticosterone and prolactin as mediators. J Physiol 1997; 498: 729-34

[13] Camargo SE, Rode Sde M, do Prado RF, Carvalho YR, Camargo $\mathrm{CH}$. Subcutaneous tissue reaction to castor oil bean and calcium hydroxide in rats. J Appl Oral Sci 2010; 18: 273-8.

[14] Borges LF, Gutierrez PS, Marana HR, Taboga SR. Picrosiriuspolarization staining method as an efficient histopathological tool for collagenolysis detection in vesical prolapse lesions. Micron 2007; 38: 580-3.

[15] Kendall AC, Whatmore JL, Winyard PG, Smerdon GR, Eggleton P. Hyperbaric oxygen treatment reduces neutrophil-endothelial adhesion in chronic wound conditions through S-nitrosation. Wound Repair Regen 2013; 21(6): 860-8.

[16] Dovi JV, He LK, DiPietro LA. Accelerated wound closure in neutrophil-depleted mice. J Leukoc Biol 2003; 73: 448-55.

[17] Butterfield TA, Best TM, Merrick MA. The dual roles of neutrophils and macrophages in inflammation: a critical balance between tissue damage and repair. J Athl Train 2006; 41: 457-65

[18] Kudaeva OT, Kolesnikova OP, Sukhenko TG, Kozlov VA. Effect of regular physical training on hemopoiesis in experimental animals. Bull Exp Biol Med 2012; 153: 217-21.

[19] Godbout C, Ang O, Frenette J. Early voluntary exercise does not promote healing in a rat model of Achilles tendon injury. J Appl Physiol (1985) 2006; 101: 1720-6.

[20] Schnoor M, Cullen P, Lorkowski J et al. Production of type VI collagen by human macrophages: a new dimension in macrophage functional heterogeneity. J Immunol 2008; 180: 5707-19. 